\title{
Dynamic Levels in Classical and Romantic Keyboard Music: Effect of Musical Mode
}

\author{
OLIVIA LADINIG ${ }^{[1]}$ \\ School of Music, Ohio State University \\ DAVID HURON \\ School of Music, Ohio State University
}

\begin{abstract}
An analysis of dynamic markings in 140 works representing three musical periods (Classical, early Romantic, late Romantic) is reported. We tested the hypothesis that minor-mode music from the Romantic period is more likely to exhibit louder dynamic levels than minor-mode music from the Classical period. This hypothesis was motivated by the theory that in the Romantic period, in addition to conveying sadness, the minor mode was more likely to be used to convey affects that are associated with higher dynamic levels, such as seriousness, passion or aggression. Our analysis showed no absolute differences regarding the notated dynamic levels of the minor-mode pieces. However, regardless of the musical mode, pieces from the earlier period exhibited higher dynamic levels than pieces from the later periods. This effect is attributable to a decrease in dynamics for major-mode pieces in later musical periods, while minor-mode pieces do not show a change in dynamics over time. Using the mean dynamic level for each period as a reference, these observations are consistent with the theory that Romantic music is more likely than Classical music to employ the minor mode to represent or convey affects that are associated with higher dynamic levels.
\end{abstract}

Submitted 2010 January 27; accepted 2010 May 3.

KEYWORDS: dynamics, minor mode, major mode, Classical music, Romantic music

RESEARCH in speech prosody has shown that sadness is associated with a number of acoustical cues including slower speaking rate, quieter voice, lower overall fundamental frequency, lower spectral centroid, and smaller pitch variance (e.g., Banse \& Scherer, 1996; Breitenstein, van Lancker \& Daum, 2001; Davitz, 1964; Fairbanks \& Pronovost, 1939; Siegman \& Boyle, 1993). Of the various factors studied, loudness has been shown to be an especially reliable indicator when assessing the emotional state of a speaker (Yildirim et al., 2004). For example, low intensity level provides a robust cue for distinguishing sadness from other affective states (Banse \& Scherer, 1996; Cowie et al., 2001; Scherer, 1986; Sobin \& Alpert, 1999).

In the domain of music, the use of the minor mode provides a strong though not infallible cue for sadness. For example, both Heinlein (1928) and Hevner (1935) showed that the use of the minor mode is typically associated with sadness for Western-enculturated listeners. More recently, a number of correlational studies of musical corpora have revealed parallels between acoustical cues for sad speech and musical features in minor-mode works. In general, minor-mode music is lower in overall pitch and exhibits smaller interval sizes (Huron, 2008), is more likely to employ dark timbres (Schutz, et al., 2008), and displays quieter notated dynamic levels (Turner \& Huron, 2008).

In the case of tempo, however, Post and Huron (2009) found no general trend: music in the minor mode is not generally slower than music in the major mode. Instead the relationship between tempo and mode was found to depend on the historical period. Minor-mode music in the Baroque and partly in the Classical periods exhibits the predicted slower tempos, but minor-mode music from the early Romantic period is associated with faster, rather than slower, tempos. In discussing this finding, Post and Huron offered a theory that might account for this observed anomaly. Specifically, they noted that music historians suggest that a literary movement known as Sturm und Drang ('storm and stress,' or 'storm and longing') influenced music making in the late Classical period, and that this became a seminal factor 
contributing to the advent of musical romanticism. Characteristic of the new musical language was a greater emphasis on passion and seriousness. A little-mentioned finding by Hevner (1935), offers some credence for such an interpretation. Not only was minor-mode music associated with sadness alone, but for Western-enculturated listeners, Hevner found that the minor mode was also evocative of mystery, passion, weirdness, seriousness, and other qualities. If it was the intention of romantic composers to convey or express an affective state such as seriousness, passion, or even aggression, research suggests that the sounds would be louder (Yildirim et al., 2004). Accordingly, we propose the following conjecture: The faster tempos associated with the minor mode in $19^{\text {th }}$ century music may have been a consequence of greater interest in the use of the minor mode to represent or convey non-sad affects. If we assume that the minor mode was also used to convey seriousness or passion, then we would expect $19^{\text {th }}$ century minormode music to exhibit higher dynamic levels compared with earlier minor-mode music.

\section{HYPOTHESIS}

In this study we propose testing the following hypothesis:

H1. Minor-mode music in the Romantic period is more likely to exhibit louder notated dynamic levels than minor-mode music in the Classical period.

The $19^{\text {th }}$ century was a period of considerable musical flux, and so there is merit to distinguishing the musical practices of the first and second halves of the century. As will be evident below, in assembling our musical sample, there were many more $19^{\text {th }}$ century works that conformed to the selection criterion than earlier works. Accordingly, we decided a priori to compare three periods, Classical (conventionally $1750-$ 1825), early-Romantic (conventionally 1825 - 1855), and late-Romantic (conventionally 1855 - 1900). We may therefore restate our hypothesis as follows:

H1a. Minor-mode music in the early and late Romantic periods are more likely to exhibit louder dynamic levels than minor-mode music in the Classical period.

In order to test this hypothesis we must first consider a potential confound, namely the overall dynamic levels in the pertinent music periods. If an examination of the dynamics of minor-mode pieces proved consistent with our hypothesis, we could not exclude the possibility that dynamics from certain periods are simply lower or higher overall and thus responsible for any observed difference. We can address this possible confound by looking at potential differences in the overall dynamic levels between historical periods, examining differences in the dynamic levels in major-mode pieces and relating them to differences in dynamics in minor-mode pieces. Using this approach we hope to circumvent this possible confound.

\section{SAMPLE}

Works were sampled from the music library at the Ohio State University. In assembling an appropriate sample, difficulties arise if one were simply to select major and minor-mode works at random. Major- or minor-mode works may be biased toward particular genres or classes of music. For example, it might be the case that minor-mode works are less likely to employ the voice-and it might also be the case that vocal music tends to be louder than works for solo instrument. If such genre-related associations exist, then a researcher might erroneously conclude that there is a direct causal connection between the minor mode and quieter dynamics when, in fact, the correlation is attributable to a third variable, such as instrumentation or musical genre. In order to avoid this hypothetical confound, a more appropriate sampling method would attempt to ensure that both the major and minor-mode works are drawn from the same musical genre or class. Accordingly, in our sampling, we made use of the '24-prelude set.' Inspired by J.S. Bach's Well-Tempered Clavier, a number of Western composers have created sets of pieces (commonly for the piano) composed in each of the 12 enharmonic major and minor keys. Typically, these sets are arranged with alternating major and minor-mode works. Some sets alternate relative major and minor keys (such as $\mathrm{C}$ major/A minor) whereas other sets alternate tonic major and minor keys (such a $\mathrm{C}$ major/C minor). 
We selected all of the sets known to us from the Classical period. This includes Muzio Clementi (born 1752, preludes published 1811), and Johann Nepomuk Hummel (born 1778, preludes composed 1814-15). In the case of the early Romantic and late Romantic periods many more sets are available. We pseudo-randomly selected four additional prelude sets: we choose two sets of works from the early Romantic period (Stephen Heller, born 1813, preludes composed 1853, and Henri Herz, born 1805, preludes composed 1830), and two from the late Romantic period (Aleksandr Scryabin, born 1872, preludes composed 1895, and Felix Blumenfeld, born 1863, preludes composed 1892). With six sets of 24 works, our nominal sample included 144 pieces, 72 in minor and 72 in major mode.

The mode for each sampled work was obvious from the ordering of the pieces within the set, or from the titles. Nevertheless we confirmed the mode for each piece by examining their initial and final harmonies as well as the key signature.

\section{METHOD}

In Western music it is common for works to depart from their nominal key through one or more modulations. In some cases, the modulation leads to a different mode; for example, a work that begins in the minor mode may contain lengthy passages in the major mode, or (less commonly) vice versa. Such changes of mode can cause confusion when coding the relationship between mode and dynamics. In order to circumvent this problem, we focused exclusively on the initial mode and the initial dynamic marking for each piece.

Notated dynamics were coded as an ordinal scale (1-6, corresponding to $p p, p, m p, m f, f$, and $f f)$. The distance between successive values may not be interpreted as equivalent in loudness difference, but the ordinal succession still allows the application of appropriate ordinal statistical methods. Four pieces (three from the Classical and one from the early Romantic period) did not exhibit any dynamic markings, reducing our sample to 140 pieces. By way of summary, for each of the 140 pieces we coded both the initial mode (major/minor) and the initial dynamic level.

\section{RESULTS}

We performed a 2 x 3 factorial analyses of variance (ANOVA), with factor A being mode (major or minor) and factor B being period (Classical, early Romantic, or late Romantic) (see Figure 1). We found no main effect for mode, suggesting that there was no general difference in the dynamics between minor- and major-mode pieces. We found a main effect of period, $F(2,134)=7.57, p=.001$, showing significantly higher dynamics for the pieces from the Classical period compared to the late-Romantic period, with nonsignificant intermediate values evident for the early-Romantic period. However, this effect was qualified by a significant interaction between mode and period, $F(2,134)=3.20, p=.044$. Pairwise comparisons (with Bonferroni alpha-level correction for multiple comparisons) showed that the effect for period was only evident in major-mode pieces, with no differences for the minor mode. Further, there are only modespecific differences in the use of dynamics in early-Romantic pieces, but not in Classical or late-Romantic pieces. 


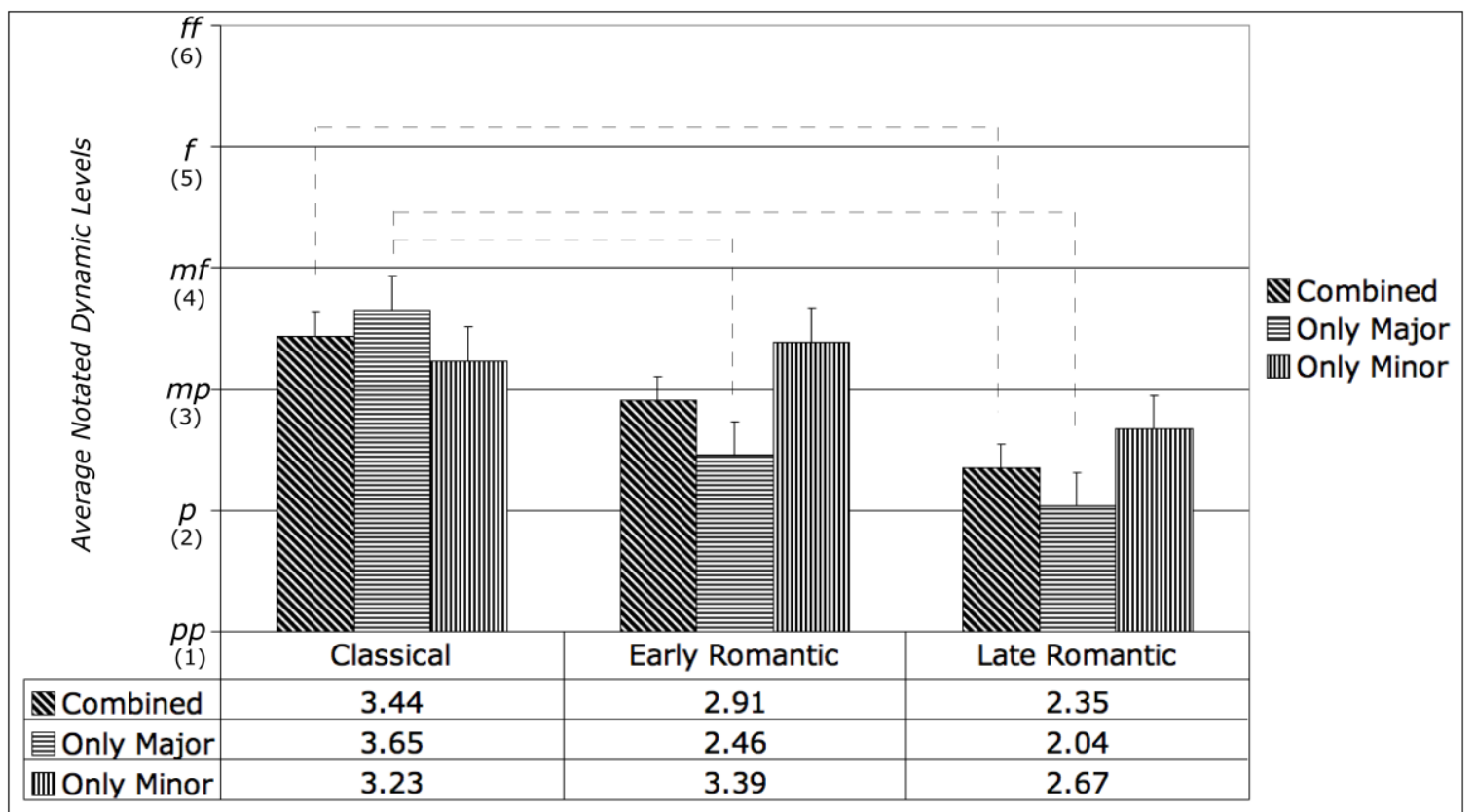

Fig. 1. Average notated dynamic levels for works in major, minor, and combined (both major and minor) modes. Results are displayed by historical period: Classical, early Romantic, and late Romantic. Dashed lines identify statistically significant differences. Error bars indicate standard errors. Ignoring mode, there is a trend evident toward quieter notated dynamics over time. No differences were found for works in the minor mode, but works in the major mode showed a decrease of notated dynamics over time. Thus, relative to the major mode, works in the minor mode became louder when moving from the Classical to the Romantic periods. This result is supportive of the view that, in the Romantic period, the minor mode was used to represent or convey affects other than just sadness - such as passion or seriousness.

\section{CONCLUSION AND DISCUSSION}

Our analysis reveals that the use of dynamics is different for the three musical periods under consideration. For pieces composed in later periods, overall notated dynamic levels are lower. When musical mode is considered, it becomes apparent that this difference stems exclusively from quieter dynamics in the major mode with no significant change in the minor mode.

At first glance, these results are not consistent with our hypothesis. According to our hypothesis we would have anticipated that starting with the early-Romantic period, there would be an increase in the use of the minor mode to express or convey non-sad affects like seriousness and passion. No differences were found for the absolute dynamic levels in minor-mode pieces over the three periods. However, another way to make some pieces sound louder is to make the rest of the repertoire quieter; in other words to change the whole referential frame and achieve loudness in relative, rather than in absolute, terms. The statistical analysis is consistent with this interpretation.

The results are consistent with at least two different interpretations. First, the results are in accord with the study by Post and Huron (2009) that showed minor-mode pieces are associated with slower tempos than the matched major-mode pieces, but that this relationship only holds for music from the Baroque period, partly for pieces from the Classical period, and is the opposite for pieces from the Romantic period. Post and Huron used an elaborate method to establish musical tempos - a method beyond the scope of this study. Nevertheless, we aimed to compare our sample to their results, and used an admittedly crude measure based on translating the notated tempo markings into bpm as labeled on a conventional metronome. Our results were partly consistent with their findings. No tempo differences between major-mode and minor-mode pieces were found for Classical and early-Romantic music. However, for late-Romantic music, minor-mode pieces received faster tempo markings than major-mode pieces, supporting the interpretation about 'a Romantic exception'.

In addition, the same results are consistent with greater use of the major mode as representing or conveying tenderness through reduced loudness (see also Zentner et al., 2008). Moreover, the results are 
consistent with the view that both interpretations may be simultaneously valid. Further research is warranted.

\section{NOTES}

[1] Please address all correspondence to: Olivia Ladinig, School of Music, 1866 College Rd., Ohio State University, Columbus, OH, 43210.

\section{REFERENCES}

Banse, R., \& Scherer, K.R. (1996). Acoustic profiles in vocal emotion expression. Journal of Personality and Social Psychology, Vol. 70, pp. 614-636.

Breitenstein, C., van Lancker, D., \& Daum, I. (2001). The contribution of speech rate and pitch variation to the perception of vocal emotions in a German and an American sample. Cognition \& Emotion, Vol. 15, pp. 57-79.

Cowie, R., Douglas-Cowie, E., Tsapatsoulis, N., Votsis, G., Kollias, S., Fellenz, W., \& Taylor, J. (2001). Emotion recognition in human-computer interaction. IEEE Signal Processing Magazine, Vol. 18, pp. 3280.

Davitz, J.R. (1964). Auditory correlates of vocal expressions of emotional meanings. In: J.R. Davitz (Ed.), Communication of Emotional Meaning. New York: McGraw-Hill, pp. 101-112.

Fairbanks, G., \& Pronovost, W. (1939). An experimental study of the pitch characteristics of the voice during the expression of emotion. Speech Monographs, Vol. 6, pp. 87-104.

Heinlein, C.P. (1928). The affective characters of the major and minor modes in music. Journal of Comparative Psychology, Vol. 8, pp. 101-142.

Hevner, K. (1935). The affective character of the major and minor modes in music. American Journal of Psychology, Vol. 47, 103-118.

Huron, D. (2008). A comparison of average pitch height and interval size in major- and minor-key themes: Evidence consistent with affect-related pitch prosody. Empirical Musicology Review, Vol. 3, No. 2, pp. 5963.

Post, O., \& Huron, D. (2009). Music in minor modes is slower (except in the Romantic period). Empirical Musicology Review, Vol. 4, No. 1, pp. 1-9.

Scherer, K. (1986). Vocal affect expression: A review and a model for future research. Psychological Bulletin, Vol. 99, pp. 143-165.

Schutz, M., Huron, D., Keaton, K., \& Loewer, G. (2008). The happy xylophone: Acoustic affordances restrict an emotional palate. Empirical Musicology Review, Vol. 3, No. 3, pp. 126-135.

Siegman, A., \& Boyle, S. (1993). Voices of fear and anxiety and sadness and depression: The effects of speech rate and loudness on fear and anxiety and sadness and depression. Journal of Abnormal Psychology, Vol. 102, pp. 430-437.

Sobin, C., \& Alpert, M. (1999). Emotion in speech: The attributes of fear, anger, sadness, and joy. Journal of Psycholinguistic Research, Vol. 28, pp. 347-365.

Turner, B., \& Huron, D. (2008). A comparison of dynamics in major- and minor-key works. Empirical Musicology Review, Vol. 3, No. 2, pp. 64-68. 
Yildirim, S., Bulut, M., Lee, C., Kazemzadeh, A., Busso, C., Deng, Z., Lee, S., \& Narayanan, S. (2004). An acoustic study of emotions expressed in speech. In Proceedings of the International Conference on Spoken Language Processing. Jeju Island, Korea, October 2004, pp. 2193-2196.

Zentner, M., Grandjean, D., \& Scherer, K.R. (2008). Emotions evoked by the sound of music: Characterization, classification, and measurement. Emotion, Vol. 8, pp. 494-521. 